\title{
Hover Position of Quadrotor Based on PD-like Fuzzy Linear Programming
}

\author{
Iswanto $^{1,2}$, Oyas Wahyunggoro ${ }^{1}$, Adha Imam Cahyadi ${ }^{1}$ \\ ${ }^{1}$ Department of Electrical Engineering and Information Technology, Universitas Gadjah Mada, Yogyakarta, Indonesia \\ ${ }^{2}$ Department of Electrical Engineering, Universitas Muhammadiyah Yogyakarta, Yogyakarta, Indonesia
}

\begin{tabular}{l}
\hline Article Info \\
\hline Article history: \\
Received Nov 27, 2015 \\
Revised Aug 5, 2016 \\
Accepted Aug 23, 2016 \\
\hline
\end{tabular}

Keyword:

Altitude control

Fuzzy linear programming

Hover position

PD-like

Quadrotor

\begin{abstract}
The purpose of this paper is to present the altitude control algorithm for quadrotor to be able to fly at a particular altitude. Several previous researchers have conducted studies on quadrotor altitude by using PID control but there are problems in the overshoot and oscillation. To optimize the control, tunning on PID algorithm must be first conducted to determine proportional and derivative constants. Hence, the paper presents altitude control modification by using PID-like fuzzy without tuning. The PID algorithm is a control algorithm for linear systems. While, system to be controlled is a non-linear, so that linearization is needed by using equilibrium. The proposed algorithm is a modification of the PID algorithm used as an altitude control which enables quadrotor to be stable when hovering. The algorithm used is not PID algorithm with tuning using fuzzy, but this is a single input single output (SISO) control PID-like fuzzy linear programming. The result of the research shows that quadrotor can hover in a rapid raise time, steady state and settling time without performing overshoot and oscillation.
\end{abstract}

Copyright (C) 2016 Institute of Advanced Engineering and Science. All rights reserved.

\section{Corresponding Author:}

Iswanto,

Departement of Electrical Engineering and Information Technology,

Universitas Gadjah Mada,

Yogyakarta, Indonesia.

Email: iswanto.s3te13@mail.ugm.ac.id

\section{INTRODUCTION}

Quadrotor is an unmanned aircraft that has four motors with BLDC type at each end and has been widely used in civilian applications, military, team search and rescue (SAR) and aerial photographs because its maneuverability, vertical takeoff and landing, hover position and its ability flying in dangerous areas [1]. However, quadrotor is a non-linear system that are very difficult to stable, therefore many researchers have conducted studies to stabilize quadrotor by controlling the fourth rotors using various methods and algorithms. The commonly used conventional controller is a proportional integral derivative (PID) which is a linear control [2].

In addition to PID control, there are modern controls used for control optimization of a quadrotor such as by Raffo et al. [3] who used $\mathrm{H} \infty$ control algorithms, and blackstepping algorithm used by Besnard et al. [4] and Zheng et al [5] to stabilize the position of the quadrotor. Model Predictive Control Algorithm and linear quadratic control is one of the modern controls used to control quadrotor by Rinaldi et al. [6] and Alexis et al. [7] respectively. In addition, the proposed method is also based on artificial intelligence to control optimization quadrotor, namely Genetic Algorithm [8], Adaptive Neuro-Fuzzy Inference System (ANFIS) [9], and fuzzy logic algorithms [10]. Such methods provide optimal results and can be implemented because they do not contain a lot of mathematical equations. 
PID Control is a linear control for linear systems such as magnetic actuator [11], Position Control [12], Trajectory Control [13], Brushless DC motors [14], and Brushed DC Motor [15] so that the PID controller has been widely used by some researchers for example by Ziegler Nichols [16], as a motor controllers since PID has a simple structure with a method of determining a common PID parameter, thus it has the ability in suppressing an interference well. However, it results in a large percentage of overshoot and the control signaling used tends to be large, which may cause saturation in the actuator. Great gesture control requires big energy in which several methods proposed to improve the control of the gesture by using hybrid control that is Particle Swarm Optimization (PSO)-based PID control [17] and fuzzy logic algorithm-based PID control [18].

Some researchers such as Zefang He and Long Zhao [19] used PID control with Ziegler-Nichols tuning used for quadrotor stability. By using feedback linearization theory, a nonlinear Quadrotor is modeled into a linear one. Other researchers such as Hassan Tanveer et al. [20] and Bolandi et al. [21] used Taylor's method for quadrotor model linerization so that the MIMO quadrotor model converted to SISO. Tuning PID using IAE was used by Bolandi et al. [21] to optimize the control of quadrotor. While auto tuning method was used by Tanveer Hassan et al. [20] to optimize PID control. Some researchers used the methods of modern control and intelligent control such as Chen et al. [22] using Neural Network for tuning the PID and Mian \& Wang [23] using backstepping for Tuning the PID.

Fuzzy linear programming has been widely used by some researchers such as Azadeh et al. [24] using it for setting in the greenhouse. Besides being used for the greenhouse, the method was used to control irrigation by $\mathrm{Lu}$ et al [25]. It can easily be applied to a linear system and has a powerful programming structure.

The purpose of this paper is to present an altitude control algorithm for quadrotor to hover stably at a certain height by using fuzzy linear programming algorithm that is PD. The systematics of this paper consists of quadrotor modeling, altitude control strategies, fuzzy controller algorithms, analyzing result and conclusion.

\section{RESEARCH METHOD}

Quadrotor system is a non-linear system that has 4 rotors at each end that can be modeled by using Eulerian angles [26]. In this paper, quadrotor is modeled by using eulerian angel thus the quadrotor system has six degrees of freedom defined by twelve states shown in Figure 1 [27]. Six out of the twelve states regulate the attitude of quadrotor system including quadrotor's Euler angles of roll, pitch, yaw i.e. $\beta_{2}=\left[\begin{array}{lll}\phi & \theta & \psi\end{array}\right]^{T}$ and angular speed of the quadrotor i.e. $\beta_{4}=\left[\begin{array}{lll}p & q & r\end{array}\right]^{T}$ on three orthogonal axes of the body. Six other states are three positions namely $\beta_{1}=\left[\begin{array}{lll}x & y & z\end{array}\right]^{T}$, and three linear speed of the center of mass of the quadrotor associated with fixed reference frame (frame earth) i.e. $\beta_{3}=\left[\begin{array}{lll}\dot{x} & \dot{y} & \dot{z}\end{array}\right]^{T}$. This quadrotor system model used 12 equations of non linear state in a global framework as $x=\left[\begin{array}{lll}x_{1} & \ldots & x_{12}\end{array}\right]^{T}$ with the state variables as $x=\left[\begin{array}{llll}\beta_{1} & \beta_{2} & \beta_{3} & \beta_{4}\end{array}\right]^{T}$.

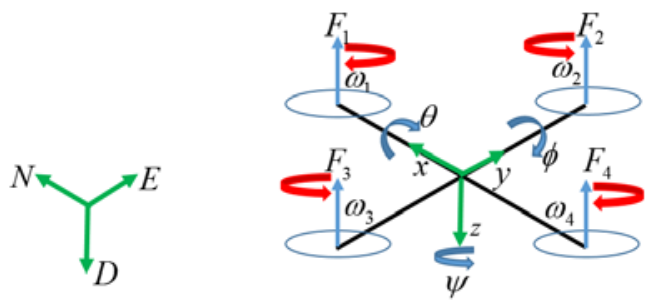

Figure 1. Quadrotor modeling with Eulerian angles

Figure 1 shows that there are two coordinate frames, one of which is the earth and the other frame is the body in the center of gravity of the quadrotor [28]. In the figure it is seen that the movement of the quadrotor by 6 degrees of freedom involving the movement of translation based on the $\mathrm{x}, \mathrm{y}$ and $\mathrm{z}$ axis. The rotation of the quadrotor is roll rotation on the $\mathrm{x}$-axis, pitch rotation on the $\mathrm{y}$-axis, and yaw rotation on the $\mathrm{z}$ axis. Each axis rotation matrix can be written as: 


$$
R(x, \phi)=\left[\begin{array}{ccc}
1 & 0 & 0 \\
0 & C \phi & -S \phi \\
0 & S \phi & C \phi
\end{array}\right] ; \quad R(y, \theta)=\left[\begin{array}{ccc}
C \theta & 0 & S \theta \\
0 & 1 & 0 \\
-S \theta & 0 & C \theta
\end{array}\right] ; \quad R(z, \psi)=\left[\begin{array}{ccc}
C \psi & -S \psi & 0 \\
S \psi & C \psi & 0 \\
0 & 0 & 1
\end{array}\right]
$$

By using the equation (1), ZYX rotation matrix is defined as

$$
R=\left[\begin{array}{ccc}
C_{\theta} C_{\psi} & S_{\phi} S_{\theta} C_{\psi}-C_{\phi} S_{\psi} & C_{\phi} S_{\theta} C_{\psi}+S_{\phi} S_{\psi} \\
C_{\theta} S_{\psi} & S_{\phi} S_{\theta} S_{\psi}+C_{\phi} C_{\psi} & C_{\phi} S_{\theta} S_{\psi}-S_{\phi} C_{\psi} \\
-S_{\theta} & S_{\phi} C_{\theta} & C_{\phi} C_{\theta}
\end{array}\right]
$$

Thus, transformation matrix obtained is as follows

$$
\left(\begin{array}{c}
p \\
q \\
r
\end{array}\right)=\left(\begin{array}{ccc}
1 & 0 & S \theta \\
0 & C \phi & -S \phi C \theta \\
0 & S \phi & C \phi C \theta
\end{array}\right)\left(\begin{array}{c}
\dot{\phi} \\
\dot{\theta} \\
\dot{\psi}
\end{array}\right)
$$

where $\mathrm{c}=\cos , \mathrm{t}=\tan$ and $\mathrm{s}=\sin$.

Based on Newton's second law of motion translational, the following equation is obtained

$$
F=m \dot{v}+(\omega \times m v)
$$

where $\omega=\left[\begin{array}{lll}\dot{p} & \dot{q} & \dot{r}\end{array}\right]^{T}$ and $V=\left[\begin{array}{lll}\dot{x} & \dot{y} & \dot{z}\end{array}\right]^{T}$.

$$
F=m\left[\begin{array}{c}
\ddot{x} \\
\ddot{y} \\
\ddot{z}
\end{array}\right]+\left(\left[\begin{array}{c}
\dot{p} \\
\dot{q} \\
\dot{r}
\end{array}\right] \times m\left[\begin{array}{c}
\dot{x} \\
\dot{y} \\
\dot{z}
\end{array}\right]\right)
$$

In Figure 1, the force acting on quadrotor is obtained formulated as

$$
F=F_{g}-F_{\text {thrust }}
$$

Substituting Newton's second law with the force acting on the quadrotor, the following equation is obtained

$$
m\left[\begin{array}{c}
\ddot{x} \\
\ddot{y} \\
\ddot{z}
\end{array}\right]+\left(\left[\begin{array}{c}
\dot{p} \\
\dot{q} \\
\dot{r}
\end{array}\right] \times m\left[\begin{array}{c}
\dot{x} \\
\dot{y} \\
\dot{z}
\end{array}\right]\right)=\left[\begin{array}{c}
0 \\
0 \\
m g
\end{array}\right]-R\left[\begin{array}{c}
0 \\
0 \\
T
\end{array}\right]
$$

Substituting the transformation matrix, the following equation is obtained

$$
m\left[\begin{array}{c}
\ddot{x} \\
\ddot{y} \\
\ddot{z}
\end{array}\right]=\left[\begin{array}{c}
-T\left(c_{\phi} s_{\theta} c_{\psi}+s_{\phi} s_{\psi}\right) \\
-T\left(c_{\phi} s_{\theta} s_{\psi}+s_{\phi} c_{\psi}\right) \\
m g-T\left(c_{\phi} c_{\theta}\right)
\end{array}\right]-m\left(\left[\begin{array}{c}
\dot{p} \\
\dot{q} \\
\dot{r}
\end{array}\right] \times\left[\begin{array}{c}
\dot{x} \\
\dot{y} \\
\dot{z}
\end{array}\right]\right)
$$

Thus the equation of linear acceleration in the $\mathrm{x}, \mathrm{y}, \mathrm{z}$ is as follows:

$$
\begin{aligned}
& \ddot{x}=-\frac{1}{m} T\left(c_{\phi} s_{\theta} c_{\psi}+s_{\phi} s_{\psi}\right)-(\dot{q} \dot{z}-\dot{r} \dot{y}) \\
& \ddot{y}=-\frac{1}{m} T\left(c_{\phi} s_{\theta} s_{\psi}-s_{\phi} c_{\psi}\right)-(\dot{r} \dot{x}-\dot{p} \dot{z})
\end{aligned}
$$




$$
\ddot{z}=g-\frac{1}{m} T\left(c_{\phi} c_{\theta}\right)-(\dot{p} \dot{y}-\dot{q} \dot{x})
$$

Based on Newton's second law of motion rotation, the following equation is obtained:

$$
\Gamma=\mathrm{I} \dot{\omega}+(\omega \times \mathrm{I} \omega)
$$

where, $\mathrm{I}$ is the moment of inertia Quadrotor as shown in following equation

$$
\mathrm{I}=\left[\begin{array}{ccc}
\mathrm{I}_{x} & 0 & 0 \\
0 & \mathrm{I}_{y} & 0 \\
0 & 0 & \mathrm{I}_{z}
\end{array}\right]
$$

while $\Gamma=\left[\begin{array}{lll}\tau_{x} & \tau_{y} & \tau_{z}\end{array}\right]^{T}$

So that the angular acceleration equation in the $\mathrm{x}, \mathrm{y}, \mathrm{z}$ is as follows:

$$
\begin{gathered}
\ddot{p}=\frac{\tau_{x}}{\mathrm{I}_{x}}-\frac{\mathrm{I}_{z}-\mathrm{I}_{y}}{\mathrm{I}_{x}} \dot{q} \dot{r} \\
\ddot{q}=\frac{\tau_{y}}{\mathrm{I} y}-\frac{\mathrm{I}_{x}-\mathrm{I}_{z}}{\mathrm{I}_{y}} \dot{p} \dot{r} \\
\ddot{r}=\frac{\tau_{z}}{\mathrm{I}_{x}}-\frac{\mathrm{I}_{y}-\mathrm{I}_{x}}{\mathrm{I}_{z}} \dot{p} \dot{q}
\end{gathered}
$$

Angular speed, $\mathrm{p}$; $\mathrm{q}$ and $\mathrm{r}$ can be obtained from the level of the Euler angles using a transformation matrix.

$$
\begin{aligned}
& {\left[\begin{array}{l}
\dot{p} \\
\dot{q} \\
\dot{r}
\end{array}\right]=W\left[\begin{array}{c}
\dot{\phi} \\
\dot{\theta} \\
\dot{\psi}
\end{array}\right]} \\
& {\left[\begin{array}{c}
p \\
q \\
r
\end{array}\right]=\left[\begin{array}{ccc}
1 & 0 & -s_{\theta} \\
0 & c_{\phi} & s_{\phi} c_{\theta} \\
0 & -s_{\phi} & c_{\phi} c_{\theta}
\end{array}\right]\left[\begin{array}{c}
\dot{\phi} \\
\dot{\theta} \\
\dot{\psi}
\end{array}\right]}
\end{aligned}
$$

So that the angular speed of roll, pitch and yaw of the quadrotor can be determined as

$$
\left[\begin{array}{c}
\dot{\phi} \\
\dot{\theta} \\
\dot{\psi}
\end{array}\right]=\frac{1}{c_{\theta}}\left[\begin{array}{ccc}
c_{\theta} & s_{\phi} s_{\theta} & c_{\phi} s_{\theta} \\
0 & c_{\phi} c_{\theta} & -s_{\phi} c_{\theta} \\
0 & s_{\phi} & c_{\phi}
\end{array}\right]\left[\begin{array}{l}
p \\
q \\
r
\end{array}\right]
$$

Thus, the roll, pitch and yaw are

$$
\begin{aligned}
& \dot{\phi}=\dot{p}+s_{\phi} t_{\theta} \dot{q}+c_{\phi} t_{\theta} \dot{r} \\
& \dot{\theta}=c_{\phi} \dot{q}+s_{\phi} \dot{r} \\
& \dot{\psi}=\frac{s_{\phi}}{c_{\theta}} \dot{q}+\frac{c_{\phi}}{c_{\theta}} \dot{r}
\end{aligned}
$$




\section{CONTROL AND STRATEGY}

Altitude control uses fuzzy linear programming, while the system will be controlled by quadrotor in a non-linear system. Therefore a linearization method for the system is needed. Non-linear models of quadrotor is then linearized at the point of balance (equilibrium), so that the system can be processed in a linear model. In conducting linearization, the point of equilibrium of the quadrotor must be determined and it can be written as $0=f(X(\alpha, \beta, \gamma, \delta))$. So that the twelve non linear equations can be written as

$$
\begin{aligned}
& \dot{x}_{1}=\dot{x}=x_{7}=0 \\
& \dot{x}_{2}=\dot{y}=x_{8}=0 \\
& \dot{x}_{3}=\dot{z}=x_{9}=0 \\
& \dot{x}_{4}=\dot{\phi}=x_{10}+s_{x_{4}} t_{x_{5}} x_{11}+c_{x_{4}} t_{x_{5}} x_{12}=0 \\
& \dot{x}_{5}=\dot{\theta}=c_{x_{4}} x_{11}+s_{x_{4}} x_{12}=0 \\
& \dot{x}_{6}=\dot{\psi}=\frac{S_{x_{4}}}{C_{x_{5}}} x_{11}+\frac{c_{x_{4}}}{C_{x_{5}}} x_{12}=0 \\
& \dot{x}_{7}=\ddot{x}=-\frac{1}{m} T\left(c_{x_{4}} s_{x_{5}} c_{x_{6}}+s_{x_{4}} s_{x_{6}}\right)=0 \\
& \dot{x}_{8}=\ddot{y}=-\frac{1}{m} T\left(c_{x_{4}} s_{x_{5}} s_{x_{6}}-s_{x_{4}} c_{x_{6}}\right)=0 \\
& \dot{x}_{9}=\ddot{z}=g-\frac{1}{m} T\left(c_{x_{4}} c_{x_{5}}\right)=0 \\
& \dot{x}_{10}=\ddot{p}=\frac{\tau_{x}}{\mathrm{I}_{x}}-\frac{\mathrm{I}_{z}-\mathrm{I}_{y}}{\mathrm{I}_{x}} x_{11} x_{12}=0 \\
& \dot{x}_{11}=\ddot{q}=\frac{\tau_{y}}{\mathrm{I} y}-\frac{\mathrm{I}_{x}-\mathrm{I}_{z}}{\mathrm{I}_{y}} x_{10} X_{12}=0 \\
& \dot{x}_{12}=\ddot{r}=\frac{\tau_{z}}{\mathrm{I}_{x}}-\frac{\mathrm{I}_{y}-\mathrm{I}_{x}}{\mathrm{I}_{z}} x_{10} x_{11}=0
\end{aligned}
$$

If it is assumed that the equilibrium point is allocated to several positions in Cartesian coordinates $(\mathrm{x} ; \mathrm{y} ; \mathrm{z})$ and at some positions of yaw angle which is defined as $x_{2}=\beta, x_{3}=\gamma$ and $x_{6}=\delta$. Thus, the value of the whole equation of state at this equilibrium point is $X(\alpha, \beta, \gamma, \delta)$, so that it can be written as $x_{1}=\alpha$, $x_{2}=\beta, \quad x_{3}=\gamma, x_{4}=0, \quad x_{5}=0, \quad x_{6}=\delta, \quad x_{7}=0, x_{8}=0, x_{9}=0, x_{10}=0, x_{11}=0, \quad$ and $x_{12}=0$. The representations of the state equation and system output are shown as follows,

$$
\begin{aligned}
& \dot{x}=A x+B u \\
& y=C x+D u
\end{aligned}
$$

where matrix A and B are obtained by using Jacobi linearization methods. Matrix A and B are derived partially to the non-linear model. 


$$
A=\left[\begin{array}{ccc}
\frac{\partial f_{1}}{\partial x_{1}} \mid X(\alpha, \beta, \gamma, \delta) & \cdots & \frac{\partial f_{1}}{\partial x_{12}} \mid X(\alpha, \beta, \gamma, \delta) \\
\vdots & \ddots & \vdots \\
\frac{\partial f_{12}}{\partial x_{1}} \mid X(\alpha, \beta, \gamma, \delta) & \cdots & \frac{\partial f_{12}}{\partial x_{12}} \mid X(\alpha, \beta, \gamma, \delta)
\end{array}\right] ; \quad B=\left[\begin{array}{ccc}
\frac{\partial f_{1}}{\partial u_{1}} \mid X(\alpha, \beta, \gamma, \delta) & \cdots & \frac{\partial f_{1}}{\partial u_{4}} \mid X(\alpha, \beta, \gamma, \delta) \\
\vdots & \ddots & \vdots \\
\frac{\partial f_{12}}{\partial u_{1}} \mid X(\alpha, \beta, \gamma, \delta) & \cdots & \frac{\partial f_{12}}{\partial u_{4}} \mid X(\alpha, \beta, \gamma, \delta)
\end{array}\right]
$$

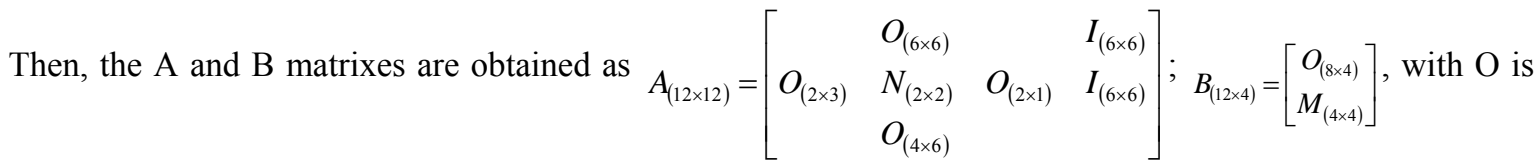
the zero matrix and I is the identity matrix. While the $\mathrm{N}$ and $\mathrm{M}$ can be defined as

$$
N_{(2 \times 2)}=\left[\begin{array}{cc}
0 & -g \\
g & 0
\end{array}\right] ; \quad M_{(4 \times 4)}=\left[\begin{array}{cccc}
-\frac{b}{m} & -\frac{b}{m} & -\frac{b}{m} & -\frac{b}{m} \\
0 & -\frac{d b}{\mathrm{I}_{x}} & 0 & \frac{d b}{\mathrm{I}_{x}} \\
\frac{d b}{\mathrm{I}_{y}} & 0 & -\frac{d b}{\mathrm{I}_{y}} & 0 \\
\frac{k}{\mathrm{I}_{z}} & -\frac{k}{\mathrm{I}_{z}} & \frac{k}{\mathrm{I}_{z}} & -\frac{k}{\mathrm{I}_{z}}
\end{array}\right]
$$

The output of the quadrotor model can be defined by the y vector as follows

$$
y=\left[\begin{array}{llll}
x & y & z & \psi
\end{array}\right]^{T}
$$

so that the $\mathrm{C}$ and $\mathrm{D}$ matrixies can be written as $C_{(4 \times 12)}=\left[\begin{array}{cc}\mathrm{I}_{(3 \times 3)} & O_{(3 \times 9)} \\ O_{(1 \times 3)} & L_{(1 \times 9)}\end{array}\right] \quad$ and $\quad D=0 \quad$ with $L_{(1 \times 9)}=\left[\begin{array}{lllllllll}1 & 1 & 1 & 0 & 0 & 1 & 0 & 0 & 0\end{array}\right]$. Thus, the linearized model of the quadrotor is

$$
\begin{aligned}
& \dot{x}=A_{12 \times 12} X+B_{12 \times 4} u \\
& y=C_{4 \times 12} X
\end{aligned}
$$

By linearization using equilibrium, the whole equations of state except $\mathrm{x}, \mathrm{y}, \mathrm{z}$, and yaw are assumed to be very small. This results in all the inputs for all four motors are assumed to have same speed. So that the MIMO system can be simplified into SISO system by using the speed of altitude change and the altitude which have the following equation

$$
\begin{aligned}
& \dot{X}_{3}=\dot{z}=X_{9} \\
& \dot{x}_{9}=\ddot{z}=g-\frac{1}{m} T\left(c_{x_{4}} c_{x_{5}}\right)
\end{aligned}
$$

The equation of the altitude system state of the quadrotor can be defined as follows

$$
\begin{aligned}
& \dot{x}_{\Phi}=A_{\Phi} x+B_{\Phi} u \\
& y=C_{\Phi} x
\end{aligned}
$$

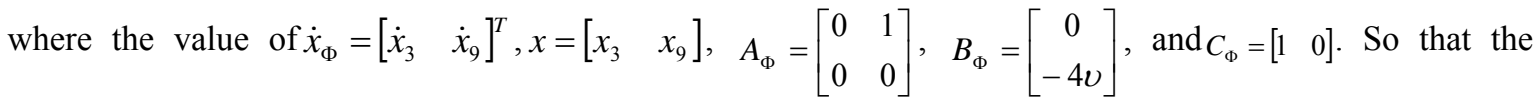
transfer function of $\mathrm{z}$ can be defined by the following equation

$$
G_{(s)}=\frac{-4 v}{s^{2}}
$$




\section{FUZZY LOGIC CONTROLLER}

Fuzzy logic algorithm is an artificial intelligence algorithms that are often used by some previous investigators to make a decision [29],[30]. The design of the altitude control of the quadrotor to maintain altitude is direct control as shown in Figure 2. The figure shows that there is altitude to control the quadrotor system. The control design is proportional control which is a linear control. Altitude control equation used in the system is proportional as seen in the following equation:

$$
G_{(s)}=\frac{-4 v K_{p}}{S^{2}+K_{p}}
$$

where $K_{p}$ is a proportional constant and $v$ is moment of inertia for each motorcycle. The stability of the system from the equation is determined by using the root locus as shown in Figure 3(A). The figure shows that there is one pair of poles on the imaginary axis in the image; that is poles located on the top side of the imaginary axis valued 3 and poles located on the bottom on the imajinear axis valued 3 . The system requires settling time and has the smallest possible overshoot. Thus it takes a large damping for the control so that the pole at the zero point of the real axis of the root locus will be shifted to the left of the real axis.

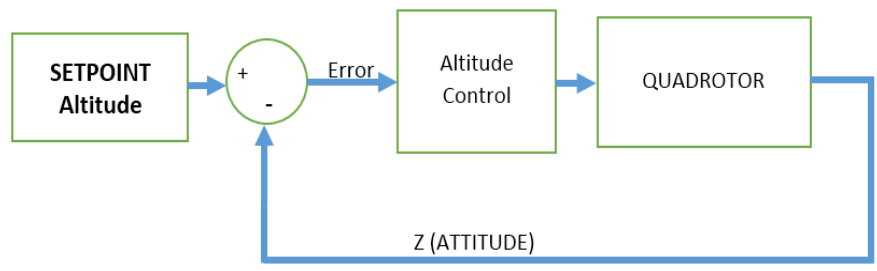

Figure 2. Block diagram of altitude control of quadrotor

Proof: by using the root locus it is seen that equation (40) has a pole value that is not on the left of the imaginary axis, then the control is unstable. The control equation was tested by using the steps shown in Figure 3(B). The figure shows that the system is not stable and there is continuous oscillation.

It proofs that Derivative in the control is necessary to become stable. Then PD control equation in the quadrotor system is as follows:

$$
G_{(s)}=\frac{K_{d}-4 v K_{p}}{S^{2}+K_{d}+K_{p}}
$$

where $K_{p}$ is a proportional constant and $K_{d}$ is a derivative constant which values are obtained by using the tuning respectively. The stability of the system in equation (42) is sought by using the root locus. Figure 4(A) shows that there is a pole located on the left of the imaginary axis that indicates the system will be stable. The equation, when tested by using steps, will perform oscillation for a short time and then will be stable as shown in Figure 4(B). It can be is seen that by using derivatives, the system will first perform oscillation, then the oscillations will be smoothed and finally it becomes stable.
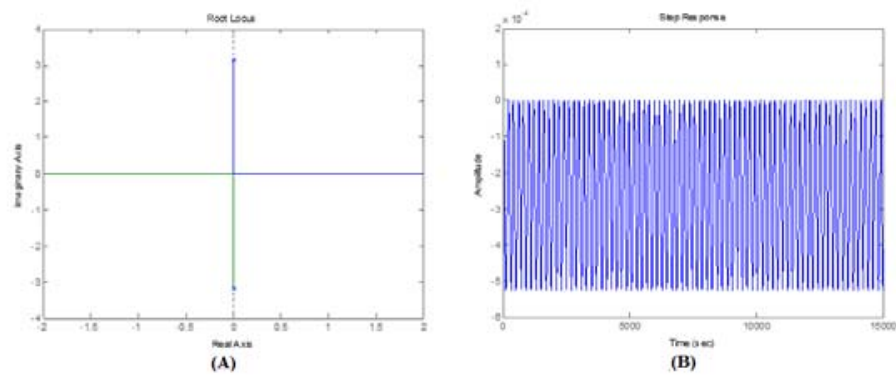

Figure 3. (A) Diagram of root locus proportional control (B) Test step for proportional control 

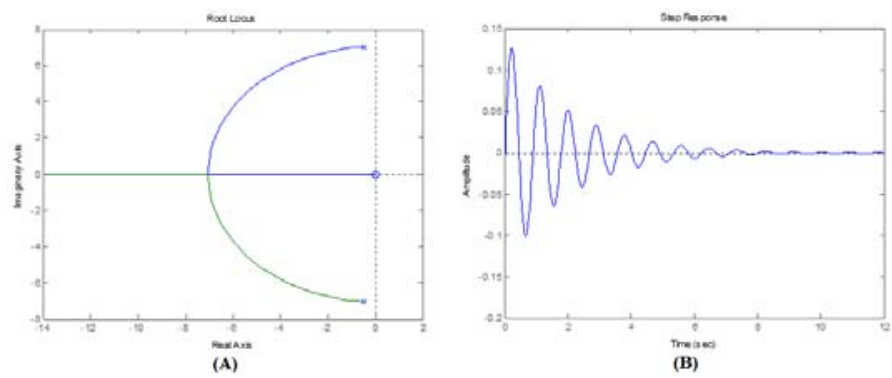

Figure 4. (A) Root locus of control that is proportional derivative. (B) Test step for proportional control of Derivative

To be optimal, a tuning to get the variable values proportional and derivative is required, so that the PID-like fuzzy linear programming is necessary for the control. This control uses SISO fuzzy of mamdani's type using three member variables for input and output that is negative small, zero, and positive small. By using three input and output variables, the rule-based is as follows

if ( $\mathrm{Z}$ is NS) then (output1 is NS)

if $(Z$ is $Z$ ) then (output1 is $Z$ )

if ( $Z$ is PS) then (output1 is PS)

Figure 5 shows altitude control using SISO control with fuzzy logic algorithms of mamdani's method. This paper presents two fuzzy controls i.e fuzzy control as proportional and derivative which have three variables of input and output set member consisting of negative, zero and positive. The output of each of the control will be summed and connected to throtell Quadrotor.

The first design conducted is fuzzy control design as propotional which has input with value error data range, so that the set variables of the input of fuzzy control as proportional has a range from -15 to 15 shown in Figure 6 (A). It is seen that the member set not only uses the up linear set and down linear set, but also uses a triangles members set. Member set is used for linear systems, while the quadrotor systems is a non-linear system, so that a linearization model is needed.

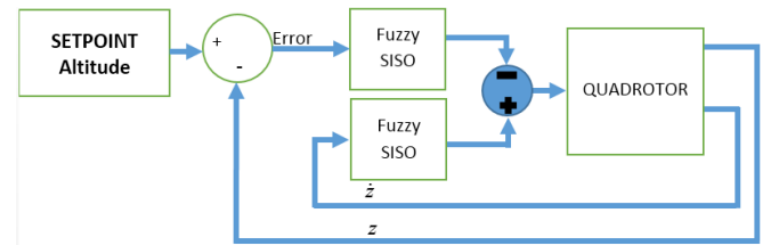

Figure 5. Block diagram of PD-like Fuzzy linier programming control

In addition to the input, fuzzy control as proportional has output in motor speeds pulse range. The determination of the output value is needed in order for the control to be optimum, so that the range for motor speed value is - Speed and + Speed shown in Figure 6 (B). The figure shows that the input value of the speed is 1200 derived from the peter Corke's model [24].

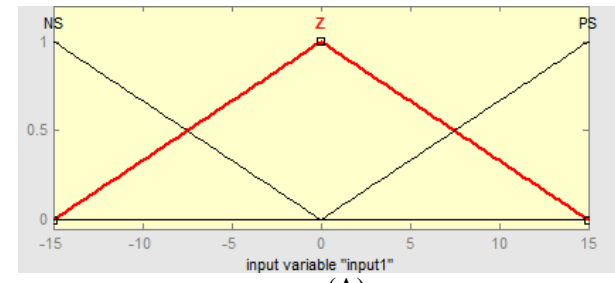

(A)

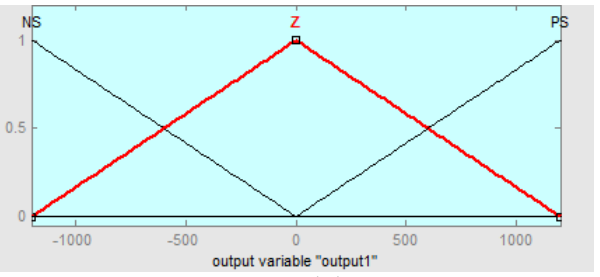

(B)

Figure 6. The set of input and output for fuzzy control as proportional 
The second design conducted is in input set design for fuzzy control as derivative shown in Figure 7. In the figure it is seen that the input set of fuzzy control as derivative has a range from $-d z$ to $d z$. The value will affect the performance of the control system. When the range value is great, the overshoot on the quadrotor can be smoothed due to the rapid acceleration values can be read by the derivative inputs, so that it will generate the output value which will result in a decrease in the proportional control value.

The design of fuzzy control output as derivatives is needed to obtain optimum altitude control of quadrotor. There are three set members that is negative small, zero, and positve small. This output variable has range which the value is obtained from half of the proportional output shown in Figure 7. The figure shows that fuzzy control output as derivatives has range value from -500 to +500 .

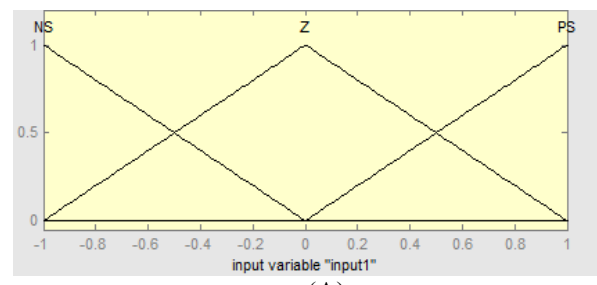

(A)

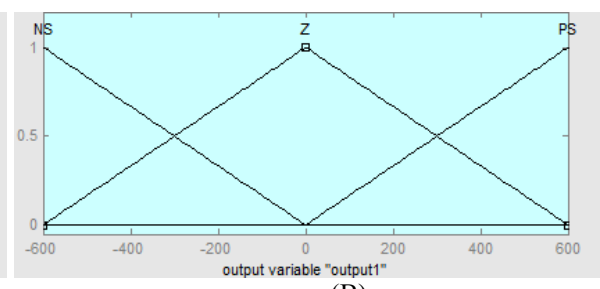

(B)

Figure 7. The set of input and output for fuzzy control as derivative

\section{RESULTS AND ANALYSIS}

Altitude control system for quadrotor hover position uses PD-like fuzzy control consisting of three parts: input, output and control. The control was tested by using peter Corke's simulation with has a specification shown in Table 1. The table shows that the quadrotor to be used for the simulation has $4 \mathrm{~kg}$ weight and has a moment of inertia $\mathrm{x}, \mathrm{y}$, and $\mathrm{z}$ of $0.0820 \mathrm{~kg} \cdot \mathrm{m}^{2}, 0.0845 \mathrm{~kg} \cdot \mathrm{m}^{2}$ and $0.1377 \mathrm{~kg} \cdot \mathrm{m}^{2}$. With the specification data provided, the stability of the quadrotor system can tested by using unit step.

Unit step is used to test the quadrotor altitude control system for 1 meter as shown in Figure 8(A). By using unit step for fuzzy logic controller algorithm the altitude control can stabilize the quadrotor, and it takes a steady state at $2.98^{\text {th }}$ second, settling time of 1.94 second, and raise time of 1.15 second which is considered short. In performing take off and hover, there are no oscillation and overshoot on the quadrotor.

Having tested by using unit step, the results shows that there is no overhoot and oscillation, the control is simulated in Peter Corke's simulator. In the simulator, firstly the parameter is set to initial position of quadrotor xy $(-1,0)$, the desired height of 8 meter, and hover position of the quadrotor xy $(-1.0)$. The experiments are performed by involving vertical wind disturbance. The simulation result shows that the quadrotor hovers at 8 meter altitude shown in Figure 8(B). There are four graphs shown in figure i.e set point, disturbance, and Error z. Altitude controller quickly stabilizes the quadrotor, and it takes a steady state at $8.384^{\text {th }}$ second, settling time of 8.975 seconds and raise time of 4.02 second with PID-Like Fuzzy algorithm but it takes a steady state at $13.39^{\text {th }}$ second, settling time of 13.9 seconds and raise time of 5.804 second with PD Loop Shaping. At $15^{\text {th }}$ second,the quadrotor is disturbed by upward vertical wind for 2 second, so that the quadrotor is shifted upward from the initial position. By using PID-like fuzzy control, it is shifted 1 meter only and return to its previous position.

Table 1. The characteristic of quadrotor

\begin{tabular}{ccr}
\hline No & Variable & \multicolumn{1}{c}{ Value } \\
\hline 1 & $\mathrm{G}$ & $9,81 \mathrm{~m} / \mathrm{s}^{2}$ \\
2 & $\mathrm{M}$ & $4 \mathrm{~kg}$ \\
3 & $\mathrm{I}_{\mathrm{x}}$ & $0.0820 \mathrm{~kg} \cdot \mathrm{m}^{2}$ \\
4 & $\mathrm{I}_{\mathrm{y}}$ & $0.0845 \mathrm{~kg} \cdot \mathrm{m}^{2}$ \\
5 & $\mathrm{I}_{\mathrm{z}}$ & $0.1377 \mathrm{~kg} \cdot \mathrm{m}^{2}$ \\
6 & $\mathrm{~B}$ & $1.2953 \times 10^{-5} \mathrm{~kg} \cdot \mathrm{m}$ \\
7 & $\mathrm{D}$ & $0.165 \mathrm{~m}$ \\
8 & $\mathrm{~K}$ & $1.0368 \times 10^{-7} \mathrm{~kg} \cdot \mathrm{m}^{2}$ \\
\hline
\end{tabular}




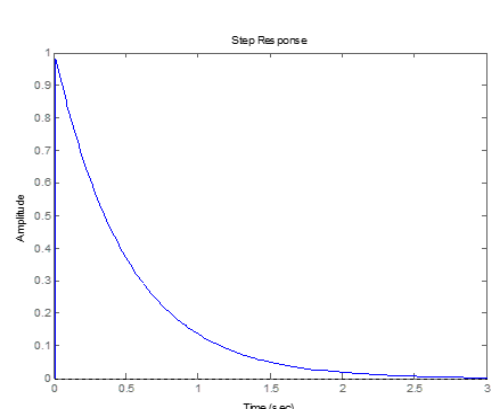

(A)

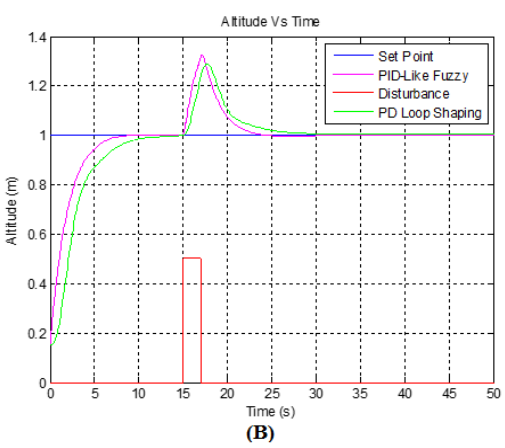

(B)

Figure 8. (A) Test step for Fuzzy control such as PD (B) Graph of quadrotor test with disturbance

\section{CONCLUSION}

Fuzzy linear programming algorithm in PD controller used for a hover position on quadrotor has been presented in this paper. This algorithm is not used as PD tuning, but it is used for controlling the altitude of the quadrotor. Based on simulation results, there are three graphs namely setpoint, altitude and disturbance which show that the quadrotor can hover without overshoot or oscillation. When the quadrotor is disturbed by vertical wind, the control can stabilize it and return it to its previous position. Thus, the fuzzy linear programming algorithms can be applied on the quadrotor which has a non-linear system.

\section{ACKNOWLEDGEMENTS}

This research was supported by PUPT Project grant from DIKTI through Research Directorate, Universitas Gadjah Mada with the contract number: 944/UN1-P.III/LT/DIT-LIT/2016, awarded to A/P Dr. Oyas Wahyunggoro.

\section{REFERENCES}

[1] N. Wen, et al., "UAV Online Path Planning Algorithm in a Low Altitude Dangerous Environment," IEEE/CAA J. Autom. Sin., vol/issue: 2(2), pp. 173-185, 2015.

[2] M. Z. Mustapa, “Altitude Controller Design for Quadcopter UAV," J. Teknol., vol/issue: 74(1), pp. 181-188, 2015.

[3] G. V. Raffo, et al., "An integral predictive/nonlinear $\mathrm{H} \infty$ control structure for a quadrotor helicopter," Automatica, vol/issue: 46(1), pp. 29-39, 2010.

[4] L. Besnard, et al., "Quadrotor vehicle control via sliding mode controller driven by sliding mode disturbance observer," J. Franklin Inst., vol/issue: 349(2), pp. 658-684, 2012.

[5] E. H. Zheng, et al., "Second order sliding mode control for a quadrotor UAV," ISA Trans., vol/issue: 53(4), pp. 1-7, 2014.

[6] F. Rinaldi, et al., "Linear quadratic control for quadrotors UAVs dynamics and formation flight," J. Intell. Robot. Syst. Theory Appl., vol/issue: 70(1-4), pp. 203-220, 2013.

[7] K. Alexis, et al., "Model predictive quadrotor control: attitude, altitude and position experimental studies," IET Control Theory Appl., vol/issue: 6(12), pp. 1812-1827, 2012.

[8] J. Yang, et al., "System Identification of Quadrotor UAV Based on Genetic Algorithm," in Proceedings of 2014 IEEE Chinese Guidance, Navigation and Control Conference, pp. 2336-2340, 2014.

[9] S. Rezazadeh, et al., "Optimal Attitude Control of a Quadrotor UAV Using Adaptive Neuro-Fuzzy Inference System ( ANFIS )," in 3rd International Conference on Control, Instrumentation, and Automation (ICCIA 2013), pp. 219-223, 2013.

[10] N. M. Raharja, et al., "Hover position quadrotor control with fuzzy logic," in International Conference on Infonnation Technology, Computer and Electrical Engineering (ICITACEE), pp. 89-92, 2014.

[11] D. Q. Truong, et al., "Development of a novel linear magnetic actuator with trajectory control based on an online tuning fuzzy PID controller," Int. J. Precis. Eng. Manuf., vol/issue: 13(8), pp. 1403-1411, 2012.

[12] N. M. Yazdani and A. Y. Seqerloo, "Performance Comparison between Classic and Intelligent Methods for Position Control of DC Motor," Int. J. Electr. Comput. Eng., vol/issue: 4(3), pp. 366-371, 2014.

[13] S. M. Mirzaei and M. H. Moattar, "Optimized PID Controller with Bacterial Foraging Algorithm," Int. J. Electr. Comput. Eng., vol/issue: 5(6), pp. 1372-1380, 2015.

[14] S. a K. Mozaffari Niapour, et al., "Brushless DC motor drives supplied by PV power system based on Z-source inverter and FL-IC MPPT controller," Energy Convers. Manag., vol/issue: 52(8-9), pp. 3043-3059, 2011.

[15] K. A. Danapalasingam, "Energy Optimization of Brushed DC Motor in Electric Power-Assisted," J. Teknol., vol/issue: 70(3), pp. 63-67, 2014. 
[16] M. A. M. Dzahir, et al., "Optimal Tuning of a Pid Controller for Emdap- Cvt Using Particle Swarm Optimization," J. Teknol., vol/issue: 75(11), pp. 135-141, 2015.

[17] H. E. a. Ibrahim, et al., "Optimal PID control of a brushless DC motor using PSO and BF techniques," Ain Shams Eng. J., vol/issue: 5(2), pp. 391-398, 2014.

[18] V. E. Omurlu, et al., "Application of Fuzzy PID Control to Cluster Control of Viaduct Road Vibrations," J. Vib. Control, vol/issue: 14(8), pp. 1201-1215, 2008.

[19] Z. F. He and L. Zhao, "A Simple Attitude Control of Quadrotor Helicopter Based on Ziegler-Nichols Rules for Tuning PD Parameters," Sci. World J., vol. 2014, pp. 13, 2014.

[20] M. H. Tanveer, et al., "Stabilized controller design for attitude and altitude controlling of quad-rotor under disturbance and noisy conditions," Am. J. Appl. Sci., vol/issue: 10(8), pp. 819-831, 2013.

[21] H. Bolandi, et al., "Attitude Control of a Quadrotor with Optimized PID Controller," Intell. Control Autom., vol/issue: 04(03), pp. 335-342, 2013.

[22] Y. Chen, et al., "Decentralized PID neural network control for a quadrotor helicopter subjected to wind disturbance," J. Cent. South Univ., vol/issue: 22(1), pp. 168-179, 2015.

[23] A. A. Mian and D. Wang, "Modeling and backstepping-based nonlinear control strategy for a 6 DOF quadrotor helicopter," Chinese J. Aeronaut., vol/issue: 21(3), pp. 261-268, 2008.

[24] A. Azadeh, et al., "A multi-objective fuzzy linear programming model for optimization of natural gas supply chain through a greenhouse gas reduction approach," J. Nat. Gas Sci. Eng., vol. 26, pp. 702-710, 2015.

[25] H. W. Lu, et al., "A Two-Step Infinite $\alpha$-Cuts Fuzzy Linear Programming Method in Determination of Optimal Allocation Strategies in Agricultural Irrigation Systems," Water Resour. Manag., vol/issue: 23(11), pp. 2249-2269, 2009.

[26] R. Mahony, et al., "Multirotor Aerial Vehicles: Modeling, Estimation, and Control of Quadrotor," Robot. Autom. Mag. IEEE, vol/issue: 19(3), pp. 20-32, 2012.

[27] N. M. Raharja, et al., "Hover position quadrotor control with fuzzy logic," in 2014 The 1st International Conference on Information Technology, Computer, and Electrical Engineering, pp. 89-92, 2014.

[28] N. M. Raharja, et al., "Altitude control for quadrotor with mamdani fuzzy model," in 2015 International Conference on Science in Information Technology (ICSITech), pp. 309-314, 2015.

[29] I. Iswanto, et al., "Path Planning Based on Fuzzy Decision Trees and Potential Field," Int. J. Electr. Comput. Eng., vol/issue: 6(1), pp. 212, 2016.

[30] I. Iswanto, et al., "Quadrotor Path Planning Based On Modified Fuzzy Cell Decomposition Algorithm," TELKOMNIKA, vol/issue: 14(2), pp. 655-664, 2016. 